disciplinas, ya sea por medio de la circulación de conceptos.. ya sea por medio de la complejización de disciplinas en campos pluri-competentes.... o .... por medio de la constitución de connarios en un sistema teórico común" (Morin, 1999 pp. 123-124).

Esta realidad - irreal, donde se ha perdido ese espacio dinámico y reconstructor llamado "nodo interdisciplinario", debe y va a cambiar. La investigación pedagógica irá en su auxilio junto a la reflexión creadora y la flexibilidad de las visiones. Están abiertas de de ellas está siendo visualizada desde uno de los espacios de formación promotor de la búsqueda de objetos perdidos, que es la Especialidad de Pedagogía Universitaria (EPU) de la PUCMM puntos de unión en la red formativa. También para descubrir e identificar nodos interdisciplinarios; me refiero a un Sistema de Indicadores de Interdisciplinariedad ${ }^{2}$ aplicable en las diferentes dimensiones y objetos curriculares: Perfil del Egresado, Plan de Estudio, Asignaturas. Las implicaciones o efectos de su aplicación trascienden estas dimensiones y objetos, para penetrar en los ámbitos estructurales, normativos y procedimentales de la academia.

2 Indicador: información que funciona como señalo o signo de caracterizazción para apreciar oreconocere el estado o grado en que se encuentra una situación oproblema
social Los indicadores pueden expresar también las tendencias de un fenómeno social. Esta definición fue tomada del Informe de un Comité de Expertos de las social. Los indicadores pueden expresar también las tendencias de un fenómeno social. Esta definición fue tomada del Informe de un Comité de Expertos de
Naciones Unidas en 1953 , creado para definir y medir los niveles de vida y designar factores concretos que intervienen en dichas condiciones (Pozas, R., 1961).

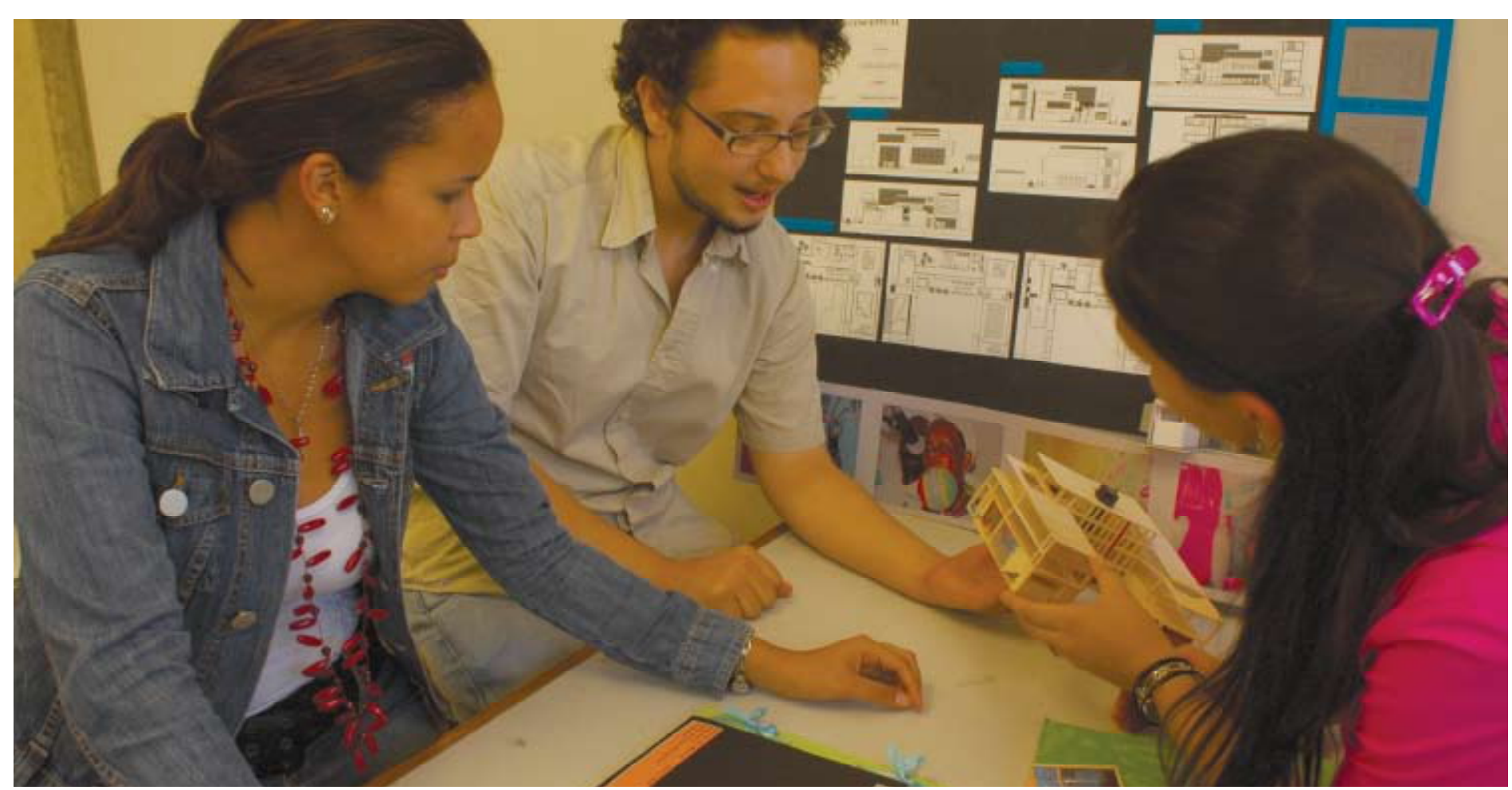

Referencias bibliográficas

Aguirre Cárdenas, J. (1999). Arquitectura y Humanidades; la Arquitectura prototipo de Interdisciplinariedad. Extraído el 8 de noviembre de 2007, de http://www.architecthum.edu.mx

áo A Berger G Brigas A. \& Michaud, G (1979) Interdisciplinariedad Problemas de la enseñanza y de la investigación en las universidades. Extraido el 8 de noviembre de 2007, de http:///biblio2.colmex.mx/bibdig/interdisciplinariedad

Morin, E. (1999). La cabeza bien puesta: repensar la reforma, reformar el pensamiento. Buenos Aires: Nueva Visión.

Pozas, R. (1961), El Desarrollo de la Comunidad: Técnicas de Investigación Social. Escuela Nacional de Ciencias Políticas y Sociales,

Universidad Nacional Autónoma de México. México: UNAM.

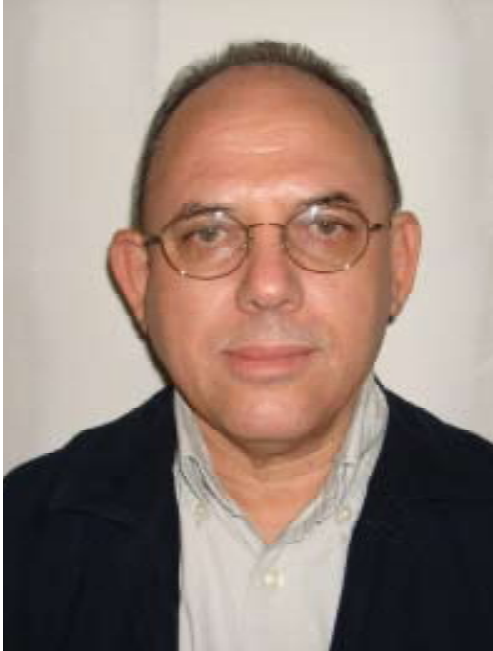

Uno de los retos del mundo actual consiste en encontrar el paso de una sociedad de la tecnología y de la información, a una sociedad de conocimiento permanente. La formulación del nuevo conocimiento radica en acompañar a las personas instituciones a surcar, desde los grandes cambios producidos por las tecnologías y la información, el camino que dignifica, a fin de que lleguen a ser lo que pueden y deben ser. Humanizar la tecnología requiere de docentes y directivos conscientes de su liderazgo en el servicio, con capacidades de gestión y con claridad sobre su propio proyec to educativo.

\section{TECNOLOGÍA, EDUCACIÓN Y HUMANISMO}

Por Serafín Coste Polanco, s.j. *

\section{Contextualización}

Eldiscurso social reciente con respecto a las ecnologias de la información y su valoración a seguido una suerte de maniqueismo, pues utilizado para hacer el bien o par hacer el mal. Es necesario hacer nuevas reflexiones ante las formas de sociedad que manera de pensar, de ser y proceder de las personas que las componen.

Uno de los factores que más inciden en estas transformaciones sociales es el producid Por las tecnologlas, la informacion y el conocimiento, pues se han ido marcando nuevas .

La sociedad del conocimiento debe prepararse $y$ capacitarse para gento debe prep y utilizar la tecnología con la finalidad responder a las demandas de desarrollo sustentable y sostenido. Además, es preciso que el vivir cotidiano no sea guiado por la tecnología sino que la tecnología sea encauzada por los seres humanos y se puedan hace aportes importantes desde ella.

En este sentido, los temas de Educación y Tecnología tienen mucho que ver con las transformaciones globales. El mundo ha ido experimentando un cambio que podemos llamar espectacular; ha sido el paso de la sociedad industrial a la sociedad de la información. Este cambio ha abrazado un nuevo modo de vida en los ámbitos de trabajo. transporte, comunicación, entre otros.
II. Finalidad y desafíos de la tecnología Uno de los grandes planteamientos actuales consiste en cómo encontrar el paso entre una sociedad de la tecnologia y la información y una sociedad de conocimiento permanente. Desde las perspectivas del presente artículo, teriber de dos vertientes.

La primera considera el aspecto de la superación del individuo en cuanto a su nivel intelectual, económico y social, que le encamina a integrarse en el mundo globalizado dentro del cauce del neoliberalismo, con todas sus
consecuencias.

La segunda, la que nos interesa realmente sin descartar los aspectos positivos de la pria, es la que abre caminos a las personas iendo que la historia; un fin que se desprende desde el momento en que Dios colocó su soplo de vida en el corazón de cada uno de nosotros.

Este es el desafío de la formación del nuevo conocimiento: acompañar a las personas e instituciones a surcar el camino que dignifica, a fin de que lleguen a ser lo que pueden y deben ser.

La tecnología es un instrumento para que podamos llegar a desarrollarnos integralmente ygarantizar la dignidad de vida en comunidad. asi como colaborar a que próximas generaciones puedan obtener ese mismo desarrollo 
desde la historia que les toque asumir. Es un medio, no un fin. Yal Para alcanzar esta sociedad deseada es necesario que el nivel decir fin, lo enfocamos desde la razón de ser y estar de cada persona, que debe sintonizar con el deseo de dignidad. La tecnologla puede ayudar a conseguir ese desear tan justo y necesario como es el corazón de cada persona.

Es necesario que existan los medios necesarios para una formación integral: académica, humana, cristiana, en y para el trabajo, que enseñe y motive la reflexión. Debe ser una formación
que produzca conocimientos, que pueda dialogar con este mundo que produzca conocimientos, que pueda dialogar con este mundo crecer enestasociedad Serequiereuna formación ymotive compartir en nuestro entorno con encuentros fraternos y de gratuidad.

Esto será posible si colocamos ternura, respeto y firmeza (esos valores que humanizan) a los avances tecnológicos del mundo de hoy, a los procesos educativos y a nuestro quehacer diario. Los valores de respeto, de tolerancia, de saber convivir en el lugar del trabajo que tenemos para existir.

No nos toca en este artículo ofrecer elementos pedagógicos o andragógicos para alcanzar ese reto. Pero sín nos toca presentar el reto en si y ayudar a crear conciencia para que cualquiera que sea la realidad donde desarrollemos nuestro quehacer formativo, no pueda pasar por alto las siguientes dimensiones: por un lado, los avances tecnológicos, conscientes de que no podemos cantinar a espaldas de los mismos $y$, por otro, que nuestros aportes deben ser desde la fraternidad y la solidaridad entre todas las personas. Esto se traduce en saber descubrir y acompañar a pulr tantas cualidades, aptitudes y deseos de nuestra gente.

\section{Ponerle corazón a la tecnología}

Ponerle corazón a la tecnología significa que ésta sea un medio para que el corazón de cada persona se desarrolle y se sustente con la reflexión que surge de toda acción formativa. Significa ciones tenemos el compromiso de convertirnos en personas de denarrollo $y$ en desarollo. El de converto tecnóng personas de descartar el desarrollo humano Es más es este último el que debe conducir al desarrollo de la tecnología La dimensión humana es previa a la tecnológica.

Cuando hablamos de la tecnología como medio, queremos decir que la misma es una herramienta que nos puede ayudar a formar mejores personas, mejores profesionales y mejores ciudadanos. Así se van creando cimientos firmes en un entorno propicio para la humanidad, donde se crezca con actitud reflexiva y crítica, donde se analice lo positivo y negativo del mismo; se haga un balance objetivo y se den respuestas a los mismos desafíos.

Estas respuestas crean dinamismos de desarrollo sustentable que impulsan hacia la sociedad que deseamos. Es una sociedad que necesita de personas con excelente formación y que sepan valorar lo humano de la vida. Siestas personas no se convierten en motores de la transformación de la misma sociedad, por más formación tecnológica que utilicemos, no llegaremos a respirar realizados.

\section{Conclusión}

Pensar en humanizar la tecnología requiere de docentes mejo preparados y remunerados, de centros educativos y aulas equipadas con los instrumentos y tecnologías adecuadas; de direcpara tivo. Además, administrativa y con claridad del proyecto educaus compromisos. Asímismo empresas que dan el salto de ir a ( aportaciones materiales e intelectuales a los procesos formativos.

Desde aquí se puede establecer el diálogo con el mundo que nos rodea, propiciar el cambio de actitudes y crear las bases de una sociedad donde las personas puedan florecer $y$ desarrollar donde se propongan y se impulsen innovaciones tecnológicas en comunidad, y esencialmente, donde los alumnos logren, con sus esfuerzos y responsabilidades compartidas, tener el gusto de vivir y sazonar con el sabor de la "vida buena" su porvenir, el porvenir de sus descendientes y de su pais.

A través de la tecnología de la comunicación somos cada vez más

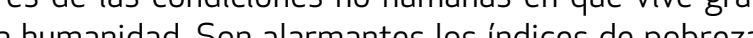
parte de la humanidad. Son alarmantes los indices de pobreza, sabiendo que el más grande de todos es el de la ignorancia. La sin las "cajas" de seguridad que sirven para guardar lo que tenemos pero no lo que somos y deseamos; sin los portones y verjas que nos encierran, nos separan y nos "cuidan" de los demás. Ojalà que el saludo mañanero y el compartir con los vecinos el café, el té, la limonada, asi como las alegrias y las penas, no se nos tercudir a la sociedad tecnificada. Ojala que no tuviéramos que Los avances tecnológicos sirven para la constucción de la vida

Generalmente, cada vez que conocemos nuevos países no podemos renunciar a la comparación de unos con otros. Al tratar de buscar lo positivo que hay en otros lugares, se ve que caemos en niveles de desarrollo sustentable. Los grandes acuerdos jorar la calidad de vida de los más pobres. Para poco sirven las grandes avenidas si no hay formación integral en las personas que las transitan. Tampoco vale de nada pretender instalaciones técnicas especializadas en los centros educativos cuando hay carencia de butacas, tizas, pizarras y borradores que faciliten proceso de enseñanza-aprendizaje. Tener permanentemente los servicios básicos de electricidad y agua potable parece ser un milagro de los generadores eléctricos y las cisternas privadas.

Desde esa comparación entre países surge la frase: "todavía en mi país se puede vivir". Ese "todavía" debemos de extirparlo. Es que nacimos para vivir. Esto significa formarnos, desarrollarnos, salir con tranquilidad por nuestras calles y caminos. Es necesario compalir lo que somos, tenemos y queremos. El desarrollo tecnologico debe estar al servicio de ese "vivin, proplciando la sociedad Formar personas para el desarrollo implica asumir la misión que nos corresponde en nuestros países: levantar las concienciasmás alládelo queprovocael subdesarrollo Debemos asumir el orden, la honestidad, la solidaridad y la justicia como normas del convivir.

Referencias bibliográficas Nacional

Escala, M. (1997). Creando Agentes de Desarrollo. Santo Domingo: CENAPEC Grass, G. (1999). Mi siglo. Madrid: Alfaguara

Pérez Esclarín, A. (1998). Educar Valores y el Valor de Educar. Caracas: San Pablo

Pérez Escalrín, A. (1999). Raíces de Fe Alegría. Caracas: Fe y Alegría

Sill, J. (1994). Exceso de Equipaje. Colombia: Norma
La tecnología es un instrumento para alcanzar la realización, el 列 a

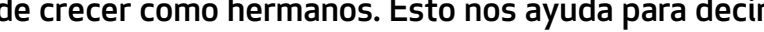
a hecio para garantizar a la sociedad la realización personal y social a los que en el futuro tomen la antorcha de la misma.

Porque soñamos con vivir mejor, siendo mejores en ese mundo tecnológico. Pensamos en que toda persona debe llegar a tener la capacidad de cuidarse a si misma y a los que le circundan, que

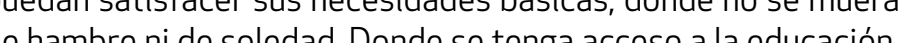
la cultura y el trabajoy desaparezcan los programas de "apoyo social".

Tenemos que poner las tecnologías al servicio de la re-creación de una sociedad donde todos nos sintamos orgullosos $y$ agradecidos de existir y vivir.

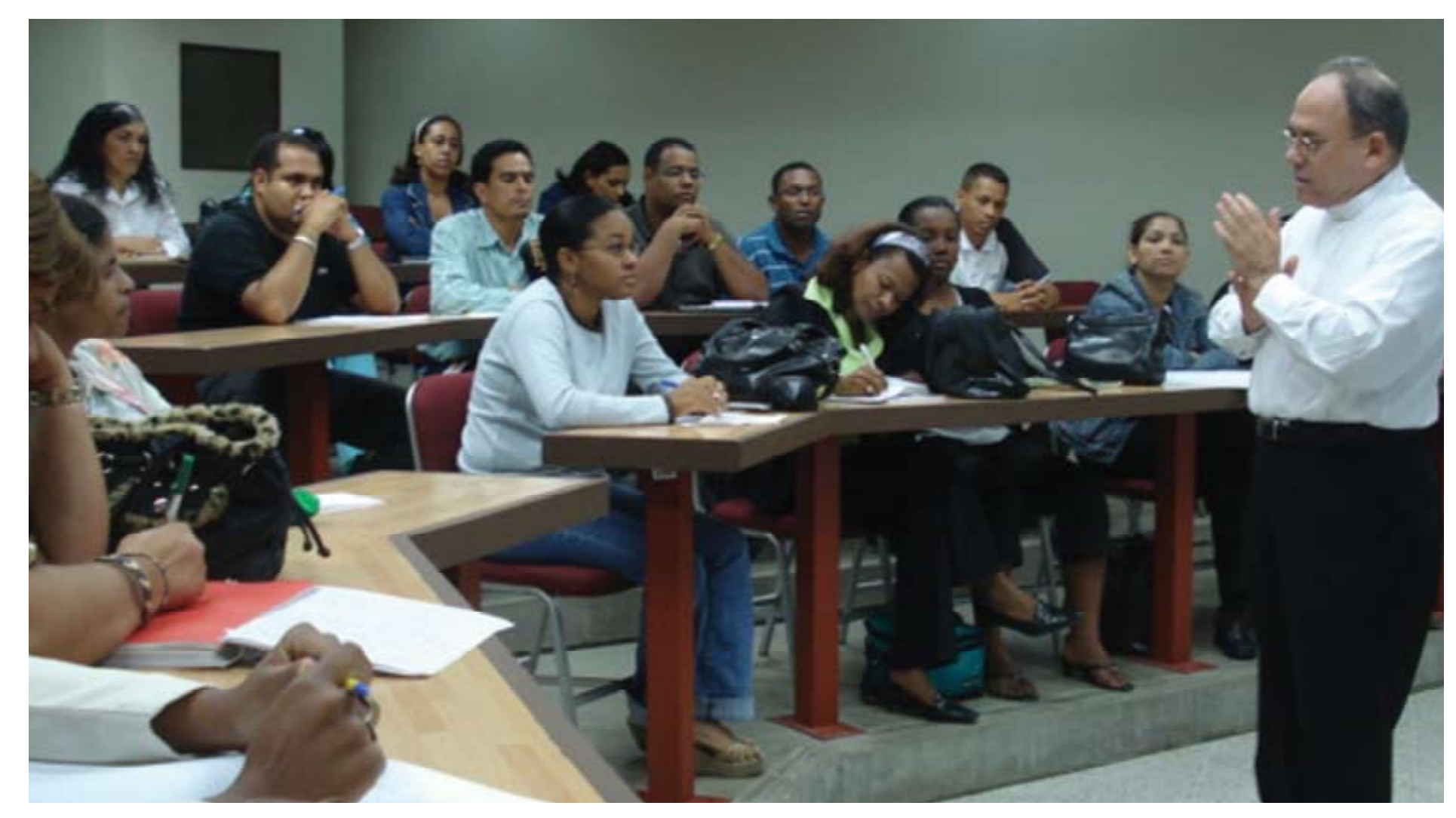

Andrade Londoño, E. (1990). Ambiente de aprendizaje para la educación en tecnología. Bogotá: Universidad Pedagógica

Gutiérrez, G. (1989). El Dios de la Vida. Lima: Centro de Estudios y Publicaciones, Insitituto Bartolomé de las casas.

Yunus, M. (1998). Hacia un Mundo sin Pobreza. Santiago de Chile: Andrés Bello 